\title{
Variação espaço-temporal das correntes de retorno em municípios da região metropolitana do Recife
}

\section{Spatial and temporal variability of rip currents in Recife metropolitan region cities}

\author{
João Carlos B. G. Maia ${ }^{\text {ac }}$, Pedro de Souza Pereira ${ }^{\text {bd }}$, Rosangela P. T. Lessa ${ }^{\text {ae }}$ \\ ${ }^{a}$ Laboratório de Dinâmica de Populações Marinhas, Departamento de Pesca e Aquicultura, Universidade Federal Rural de \\ Pernambuco, 'baboratório de Oceanografia Geológica, Departamento de Oceanografia, Centro de Tecnologia e Geociências, \\ Universidade Federal de Pernambuco \\ cmaiapemaia@hotmail.com, ${ }^{\mathrm{d}}$ psppraias@gmail.com, ${ }^{\mathrm{e}} \mathrm{rptlessa@gmail.com}$
}

\begin{abstract}
Resumo
Correntes de retorno são correntes por onde água retorna e flui rapidamente de maneira perpendicular a praiana direção do mar. Ocorrem em diversos tipos de praia, e são uma das maiores ameaças naturais a banhistas que são carregados por elas para águas mais profundas em pouco tempo, podendo causar afogamentos. Este trabalho teve como objetivo realizar o mapeamento espaço-temporal das correntes de retorno encontradas nas praias dos municípios de Cabo de Santo Agostinho, Jaboatão dos Guararapes e Recife do estado de Pernambuco. Para isso foram realizadas análises quali-quantitativas através de imagens aéreas oblíquas, obtidas por meio de sobrevoo de helicóptero e imagens de satélite, que levaram em conta, entre outras coisas, a existência de aberturas nos recifes, atividade da zona de surfe, presença de embaiamento na praia e ocorrência de plumas de sedimento. No total foram encontradas 77 correntes de retorno e 08 Indicadores de Circulação Transversal (ICT). As análises demonstraram que o maior número de fenômenos oceanográficos encontrados foram corrente de retorno móvel, 53 no total, seguidas das correntes de retorno fixas e dos ICTs. O município que mais apresentou a existência de correntes de retorno foi Cabo de Santo Agostinho, para o qual foram contabilizadas 37 correntes de retorno. Logo depois vem o município de Recife e Jaboatão com números absolutos de 15 e 09, respectivamente. Esses dados demonstram uma presença marcante de tais perigos praiais para os banhistas que frequentam as praias do estado de Pernambuco.
\end{abstract}

Palavras-chave: correntes de retorno fixas; correntes de retorno móveis; recifes

\begin{abstract}
Rip currents are currents where the water returns and flow rapidly and perpendicular to the beach toward the sea. Occur in many types of beach, and are one of the biggest natural threat to bathers that are loaded by them for deeper water in a short time, may causing drowning. The study aimed to achieve the spatial-temporal mapping of rip currents found at the beaches of the municipalities of Cabo de Santo Agostinho, Jaboatão dos Guararapes and Recife in Pernambuco state. For this qualitative and quantitative analyzes were made using oblique aerial images, obtained through helicopter over flight and satellite images, which took into account, among other things, the existence of openings in the reefs, activity in the surf zone, presence of embayment on the beach and the occurrence of sediment plumes. In total 77 rip currents and 08 Transverse Circular Indicators (TCI) were found. The analyzes showed that the greatest number of oceanographic phenomenon's were found was transient rip currents, 53 in total, followed by the rip currents fixed and TCIs. The municipality that showed the greatest number of rips was Cabo de Santo Agostinho, for which 37 rip currents were recorded. Soon after comes Recife and Piedade with absolute numbers of 15 and 09, respectively. These data demonstrate a strong presence of such beach hazards for swimmers that frequenting the beaches of Pernambuco state.
\end{abstract}

Key words: permanent rip current; mobile rip currents; reefs.

\section{Introdução}

A praia, nos tempos modernos, tem sido considerada pelo homem como um ambiente de lazer e diversão. Seu contato com este ambiente passou a ser maior devido ao crescimento populacional das cidades costeiras e de suas possibilidades de acesso a esses locais, passando a ser o local de diversão mais popular no mundo todo. Porém, quando as praias passam a agrupar um fluxo muito grande de pessoas tendem a oferecer perigos associados ao banho de mar (Mocellin 2006). Perigos Praiais são elementos do ambiente que 
Papel da praia na proteção da costa e as alterações oceanográficas em diferentes escalas temporais

propiciam aos seus usuários riscos, podendo causarlhes danos físicos e/ou até óbitos. Os perigos podem ser físicos, a exemplo de rochas, correntes de retorno fixas ou móveis, topografia, buracos entre outros. E biológicos, como incidentes com tubarões ou existência de cnidários. Sendo considerados, ainda, como permanentes e/ou não permanentes (Pereira et al. 2003). Nesse âmbito vem se destacar o papel das correntes de retorno como um dos principais perigos aos usuários de praias.

Correntes de retorno, ou, abreviadamente, CRs, ou na língua inglesa, “rip currents”, são correntes de água que fluem de maneira rápida, até $3 \mathrm{~m} / \mathrm{s}$, e perpendicular a praia se movendo na direção do mar, em alguns casos além do limite da zona de surfe. Ocorrem em quase todos os tipos de praia, e são uma das maiores ameaças naturais a banhistas, que são carregados por elas para águas mais profundas, em um curto intervalo de tempo, podendo resultar em afogamentos (Pereira et al. 2003, MacMahan et al. 2006, Trujillo \& Thurman 2011).

A conformação das correntes de retorno, conforme a figura 1 consiste basicamente da chegada das ondas à zona de arrebentação, ou zona de surfe, onde elas acabam arrebentando gerando um fluxo mais fraco, amplo e vizinho a corrente de retorno o qual mantém as correntes alimentadoras direcionadas ao longo da costa. Como o fluxo é constante a água que flui através dessas correntes alimentadoras continua seu caminho criando uma corrente na porção do pescoço, sendo este um canal. Essa corrente retorna a água no sentido do mar, onde o fluxo confinado tende a dissipar-se (na porção conhecida como cabeça) próximo ou longe da costa (MacMahan et al. 2006).

Dalrymple et al. (2011) citam, ainda, que dentro da zona de surfe a porção da circulação da corrente de retorno, referente ao fluxo de água direcionado mar a fora, é vista através de uma região mais escura, associada ao canal mais profundo e região sem quebra de ondas, que intercala porções onde se tem intenso regime de quebra das mesmas. Frequentemente essas características são utilizadas para identificação de correntes de retorno.

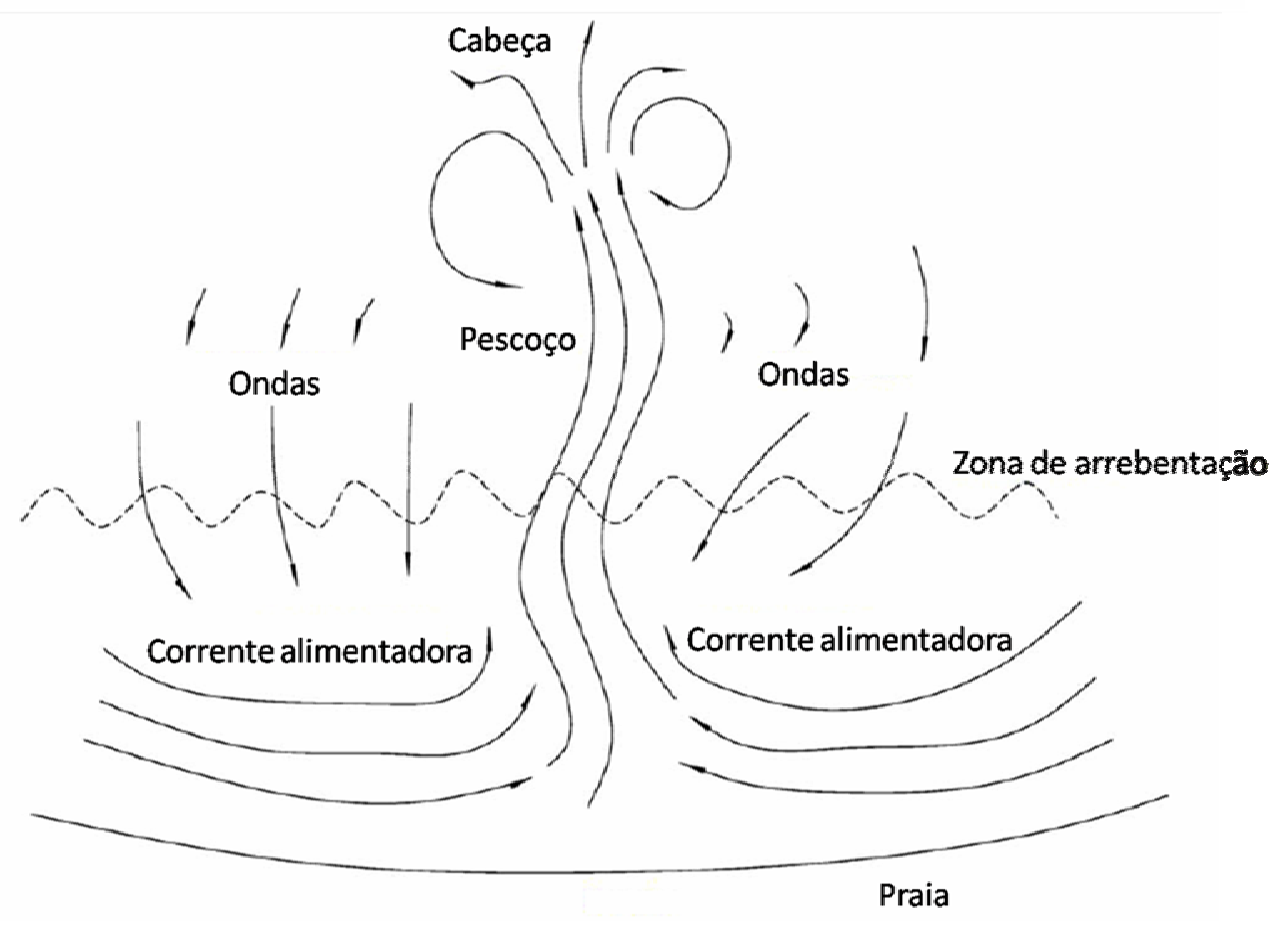

Figura 1: Esquema de uma corrente de retorno. Modificado de Shepard et al. (1941).

\section{Morfodinâmica praial}

Um sistema praial apresenta características de acordo com sua dinâmica costeira e morfologia. Estudos nesse campo conseguem associar essas duas esferas gerando descrições completas do sistema que incluem, entre outras áreas, a zona de surfe (Calliari et al. 2003). A zona de surfe é a região onde ocorre a maior parte da dissipação da energia das ondas, e onde, também, acontece grande parte dos processos que interferem na modificação morfológica e hidrodinâmica de uma praia. Ela é definida como a área que fica entre a face da praia e a arrebentação (Masselink \& Short 1993) e onde a maior parte dos banhistas é encontrada.
Wright \& Short (1984) propuseram um modelo de classificação para praias arenosas dominadas por ondas que definiu estágios extremos, dissipativo e refletivo, e estágios intermediários - Banco e Cavas Longitudinais, Banco e Praias Rítmicos (BPR), Banco Transversal e "Rips" (BTR) e Terraço de BaixaMar (TBM). Esses estágios são caracterizados por condições ambientais, de ondas, tamanho de sedimento, presença ou não de feições como cavas, bancos de areia e cúspides que, por sua vez, irão indicar e/ou influenciar as correntes de retorno com relação a sua existência e intensidade. 
Papel da praia na proteção da costa e as alterações oceanográficas em diferentes escalas temporais

\section{Arcabouço teórico}

Estudos sobre correntes de retorno vêem sendo realizados desde a década de 30 e em várias localidades do mundo como nos EUA, Japão, Dinamarca, Israel e inúmeros outros países (Shepard 1936, Sasaki \& Horikawa 1978, Aagaard et al. 1997, Bowman et al. 1988). As análises que constituíram estes estudos trataram das mais diversas influências que atuam sobre a corrente de retorno tais como: relação entre batimetria e linha de costa, influência das ondas, características das correntes, modulação do gradiente de pressão e assim por diante, sendo que na última década houve um aumento significativo desses estudos possibilitando o avanço sobre o conhecimento acerca desse fenômeno oceanográfico (MacMahan et al. 2006).

Através de uma breve revisão sobre os estudos já desenvolvidos sobre correntes de retorno, temos como primeiros trabalhos os de Shepard (1936), Shepard et al. (1941) e Shepard \& Inman (1950), onde se encontram informações referentes a descrição clássica das CRs, identificação visual do fenômeno, relação entre a batimetria, influências qualitativas entre maré e ondas e pulsação das correntes de retorno. Cabe salientar aqui, também, os trabalhos de Munk (1949a,b) os quais apresentaram a primeira tentativa de explicar as correntes de retorno, baseado no argumento cinemático de que o transporte de massa para a costa, pelas ondas sobre um banco de areia, tem saída mais eficiente por meio do canal que forma a corrente de retorno.

Estudos que se referem a mapeamentos espaçotemporal das correntes de retorno são ainda escassos. Dentre estes, os trabalhos já desenvolvidos e que merecem citação, incluem o de Short (1985), onde foram apresentados resultados de 19 meses de observação visual de CRs com relação a espacialização e permanência dessas. Outra pesquisa de grande relevância é a de Short \& Brander (1999), dentro da qual foram mostrados dados sobre densidade das correntes de retorno obtidas por meio de fotografias aéreas de diversas praias mundo a fora. A pesquisa de Holman et al. (2006), que analisou quatro anos de imagens diárias de longa exposição da praia de Palm Beach, Australia, e um total de 5.271 canais de retorno em 782 dias. Um dos trabalhos mais recentes que se pode salientar é a pesquisa de Murray et al. (2013) que estudaram o comportamento de correntes de retorno transitórias utilizando imagens de vídeo da praia de Gold Coast, Austrália.

\section{Justificativa}

Como constatado ao longo dessa introdução as correntes de retorno têm elevada importância no âmbito da segurança no banho de mar. Segundo a Organização Mundial de Saúde (OMS) cerca de 0,7\% das mortes pelo mundo tem como causa o afogamento (Szpilman 2012), e dentro dessa porcentagem as correntes de retorno surgem como uma das maiores causas naturais de afogamentos a frequentadores de praias. No Brasil as CRs tiveram um percentual de 86\% de ocorrência na praia do Futuro, em Fortaleza, (Albuquerque et al. 2010) e 66\% nas praias do litoral norte de Santa Catarina (Mocellin 2006). Por conta disso, o presente estudo assume grande importância uma vez que trabalhos nessa ótica não são encontrados em números expressivos para as praias brasileiras principalmente para a região nordeste e mais especificamente Pernambuco. As correntes de retorno estudadas no litoral brasileiro limitam-se, em grande parte, as praias mais ao sul do país. Tal fato contribui para uma maior desinformação da população a cerca do assunto que acaba por potencializar o número de banhistas que são vítimas de tal perigo.

\section{Objetivo}

A existência de correntes de retorno é um fator inerente a diversos tipos de praias e para a zona costeira brasileira isso não é diferente. Especificamente No estado de Pernambuco, pode-se mencionar que é encontrada uma alta diversidade de tipologias praiais, como por exemplo, praias protegidas por recifes, praias expostas e abrigadas, praias intensamente urbanizadas. Por esse motivo, o presente estudo objetiva realizar o mapeamento espaço-temporal das correntes de retorno encontradas nas praias dos municípios de Cabo de Santo Agostinho, Jaboatão dos Guararapes e Recife, todos integrantes da Região Metropolitana do Recife, incrementando o conhecimento a cerca desse fenômeno presente nas praias brasileiras.

\section{Área de estudo}

A localização geográfica da capital do estado Pernambucano, Recife, que conta com as praias de Boa Viagem e Pina, tendo no total uma faixa de $8,5 \mathrm{~km}$ de praia, é entre a latitude $8^{\circ} 04^{\prime}$ ' $03^{\prime}$ 'S e longitude $34^{\circ} 55^{\prime}$ 00'’W. Limita-se, ao norte, com Olinda e, ao sul, com Jaboatão dos Guararapes; a leste com o Oceano Atlântico e a oeste com Camaragibe e São Lourenço da Mata, o Parque dos Manguezais, o canal do Rio Jordão e Setúbal (Aureliano 2000). Também possui uma forte indústria turística que recebeu, segundo a Empresa de Turismo de Pernambuco (EMPETUR), no ano de 2013, mais de cinco milhões de turistas, fato que ajuda para o grande número de frequentadores nas praias. O município de Jaboatão dos Guararapes está localizado na coordenada de latitude $08^{\circ} 06^{\prime} 46^{\prime \prime}$ S e longitude $35^{\circ} 00^{\prime} 53^{\prime}$ O. Conta com as praias de Piedade, Barra de Jangada e Candeias que juntas ocupam uma faixa de litoral com 5,5 km de extensão. Este município se limita ao norte com São Lourenço da Mata e Recife, ao sul com o Município do Cabo de Santo Agostinho e a oeste com o Município de Moreno. Sua Sede principal fica distante de Recife cerca de $20 \mathrm{~km}$ e os rios que compõem a principal rede de drenagem da área são o Jaboatão e o Pirapama, ambos perenes (Assunção et al. 1997, Borba 1999). O município do Cabo de Santo Agostinho situa-se na porção sul da Região Metropolitana do Recife (RMR), distando $41 \mathrm{~km}$ da capital. É representado, nesse estudo, pelas praias de 
Papel da praia na proteção da costa e as alterações oceanográficas em diferentes escalas temporais

Gaibu, Enseada dos Corais, Itapuama, Pedra do Xaréu e Paiva. Seus limites são ao norte com os municípios de Moreno e Jaboatão dos Guararapes, a oeste com os municípios de Escada e Vitória de Santo Antão e a sul com o município de Ipojuca. Suas coordenadas geográficas são: $8^{\circ} 17^{\prime} 15^{\prime \prime}$ de latitude sul e $35^{\circ} 02^{\prime} 00^{\prime \prime}$ de longitude oeste (Assis 1999).

No que tange o regime de ventos e correntes o estado de Pernambuco possui um sistema de circulação costeira regulado pela direção e intensidade dos ventos, que por sua vez são balanceados pelo movimento latitudinal do centro de alta pressão do Atlântico Sul. Ventos sudestes, que são mais intensos e freqüentes e ocorrem no verão, impõem correntes no sentido sulnorte. Já ventos de nordeste, mais fracos, com menor frequência e com ocorrência no inverno, originam correntes de norte-sul (Lira et al. 2010).

Segundo Manso et al. (2006), Pernambuco possui uma zona costeira que corresponde a $4 \%$ de seu território e que abriga 43,8\% da população. O litoral é delimitado e setorizado de acordo com a situação geográfica e política de cada município. Os 21 municípios que compõem essa faixa estão distribuídos dentro dos 187 km de extensão litorânea delimitada, ao norte, por Goiana e, ao sul, por São José da Coroa Grande. Do total dos municípios foram determinadas para este estudo as praias de Gaibu, Enseada dos Corais, Pedra do Xaréu, Itapuama, Paiva, Piedade, Boa Viagem e Pina, que pertencem, respectivamente, aos municípios de Cabo de Santo Agostinho, Jaboatão dos Guararapes e Recife (figura 2).

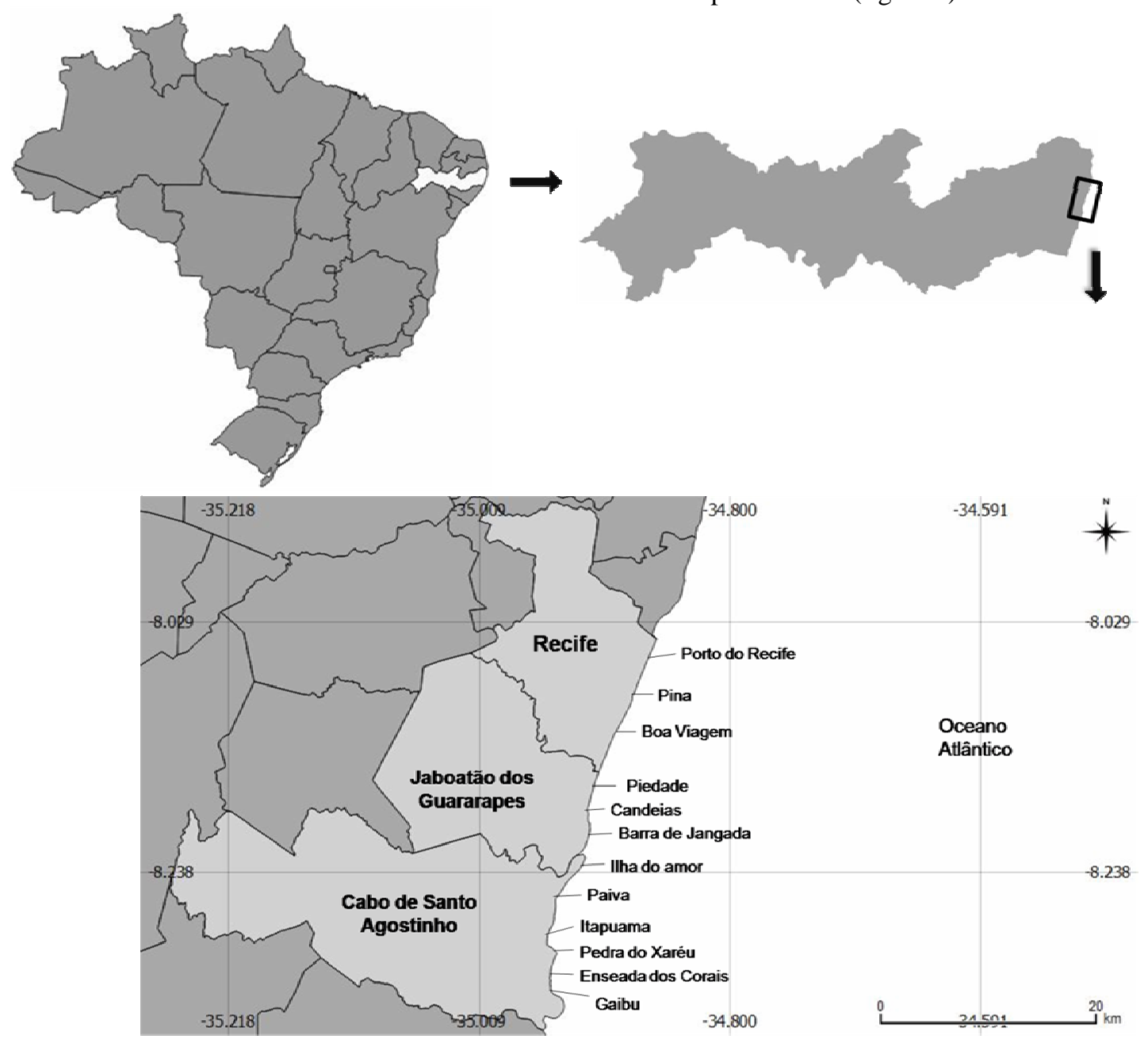

Figura 2: Área de estudo.

\section{Metodologia}

\section{Obtenção das imagens}

O estudo teve como base a identificação das correntes de retorno nas praias de Gaibu, Enseada dos Corais, Itapuama, Pedra do Xaréu, Paiva, Piedade, Boa Viagem e Pina por meio de imagens aéreas oblíquas, obtidas através de sobrevôos nos horários de preamar e baixa-mar, respectivamente, a bordo do helicóptero da
Secretaria de Defesa Social do Estado de Pernambuco (SDS). Bem como, por meio de imagens de satélites disponibilizadas pelo Laboratório de Cartografia Costeira da Universidade Federal de Pernambuco (LACCOST-UFPE) e, ainda, através de imagens gratuitas disponíveis no software Google Earth ${ }^{\circledR}$ conforme tabela 1. Dentre as imagens analisadas, as do Quickbird e Geoye apresentam alta resolução na escala de 1:10.000, e as demais apresentaram escalas variadas. 
Papel da praia na proteção da costa e as alterações oceanográficas em diferentes escalas temporais

Tabela 1: Fonte das imagens utilizadas para identificação e mapeamento das correntes de retorno.

\begin{tabular}{|c|c|c|c|}
\hline Local & Imagem & Fonte & Data \\
\hline Pina/Boa Viagem/Piedade & Quickbird & LACCOST & 2005 \\
\hline Pina/Boa Viagem/Piedade & Geoeye & LACCOST & 2011 \\
\hline Pina/Boa Viagem/Piedade & Digital Globe & Google Earth (C) & 26/01/2007 \\
\hline Pina/Boa Viagem/Piedade & Digital Globe & Google Earth (C) & 07/07/2009 \\
\hline Pina/Boa Viagem/Piedade & Digital Globe & Google Earth (C) & $24 / 11 / 2009$ \\
\hline Pina/Boa Viagem/Piedade & Digital Globe & Google Earth (C) & $18 / 06 / 2010$ \\
\hline Pina/Boa Viagem & Digital Globe & Google Earth (C) & $29 / 06 / 2011$ \\
\hline Pina/Boa Viagem & Digital Globe & Google Earth (C) & $27 / 01 / 2013$ \\
\hline Piedade & Digital Globe & Google Earth (C) & $22 / 04 / 2013$ \\
\hline Boa Viagem/Piedade & Digital Globe & Google Earth (C) & $25 / 04 / 2013$ \\
\hline Pina/Boa Viagem & Digital Globe & Google Earth C & 09/06/2013 \\
\hline Cabo de $\mathrm{St}^{\circ}$ Agostinho/ Recife/ Jaboatão dos Guararapes & Imagens aéreas oblíquas & Sobrevôo de helicóptero & $16 / 10 / 2013$ \\
\hline Cabo de $\mathrm{St}^{\circ}$ Agostinho/ Recife/ Jaboatão dos Guararapes & Imagens aéreas oblíquas & Sobrevôo de helicóptero & 23/10/2013 \\
\hline
\end{tabular}

\section{Análises das imagens}

As imagens aéreas obtidas foram processadas na forma de uma pré-visualização das mesmas para identificar quais imagens se adequavam aos objetivos deste estudo, uma vez que em algumas imagens o fenômeno oceanográfico não foi capturado por inteiro ou a visibilidade era baixa ou havia efeito de reflexo da água que dificultava a análise. Após essa etapa, foi realizada uma análise criteriosa de todas as imagens, de forma qualitativa, onde foram estabelecidos parâmetros a serem observados para a identificação das correntes
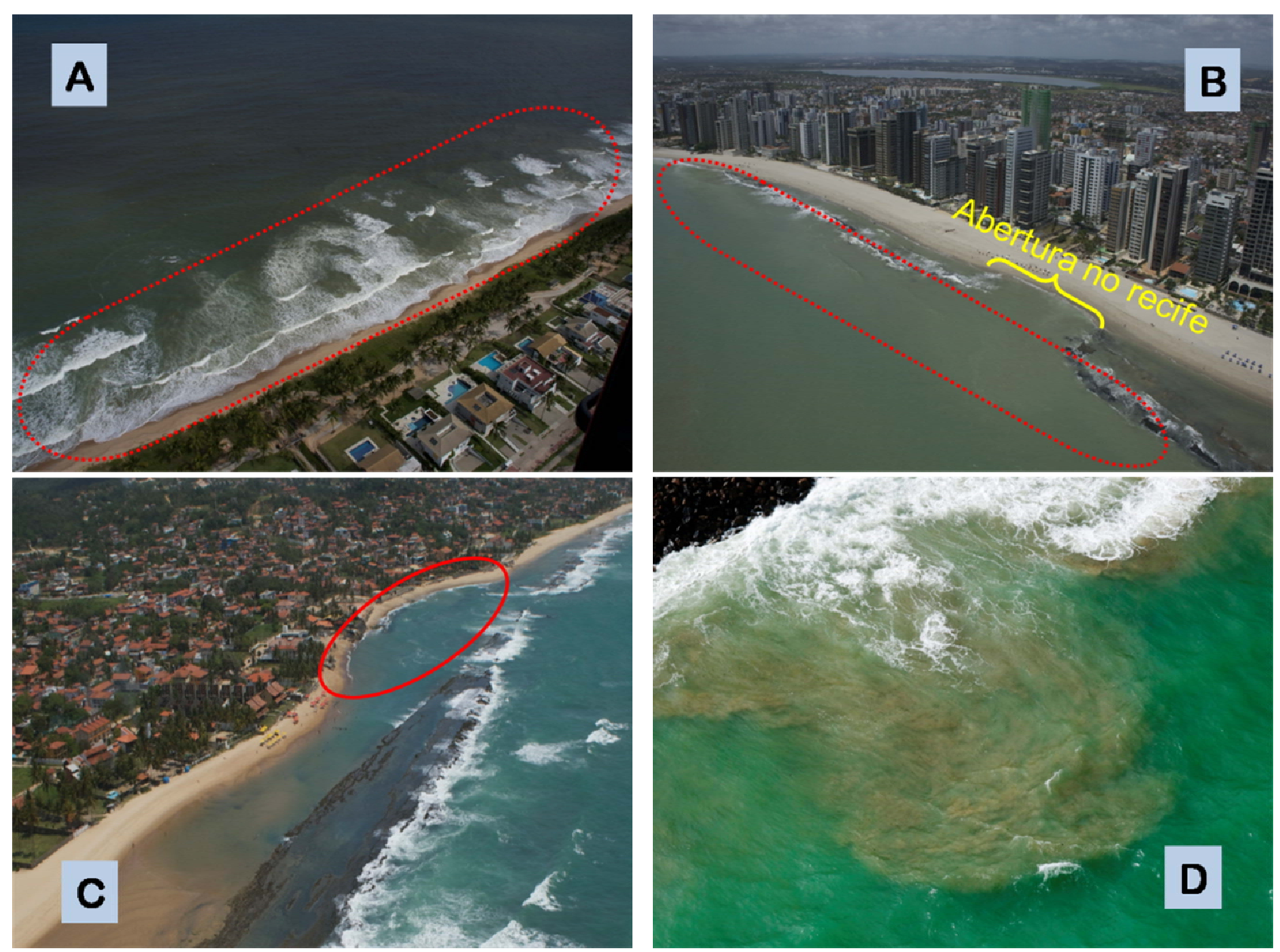

Figura 3: (A) Exemplo de zona de surfe ativa; (B) Em vermelho: zona de surfe inativa. Em amarelo: abertura no recife; (C) Presença de embaiamento; (D) Plumas de sedimento visíveis nas fotografias aéreas.

de retorno. Tais parâmetros englobaram aberturas nos recifes; a zona de surfe (ativa ou inativa); a presença de embaiamentos na praia; a ocorrência de plumas de sedimento - dentro e fora da zona de surfe - (figura 3). A classificação quanto ao tipo de corrente de retorno móvel ou fixa - que foi complementada pelo perfil morfológico associado a ela (canal profundo, rochas ou recifes); o estágio da maré; as estruturas das CRs (alimentador, pescoço e cabeça); a arrebentação das ondas e a direção das correntes (figura 4).

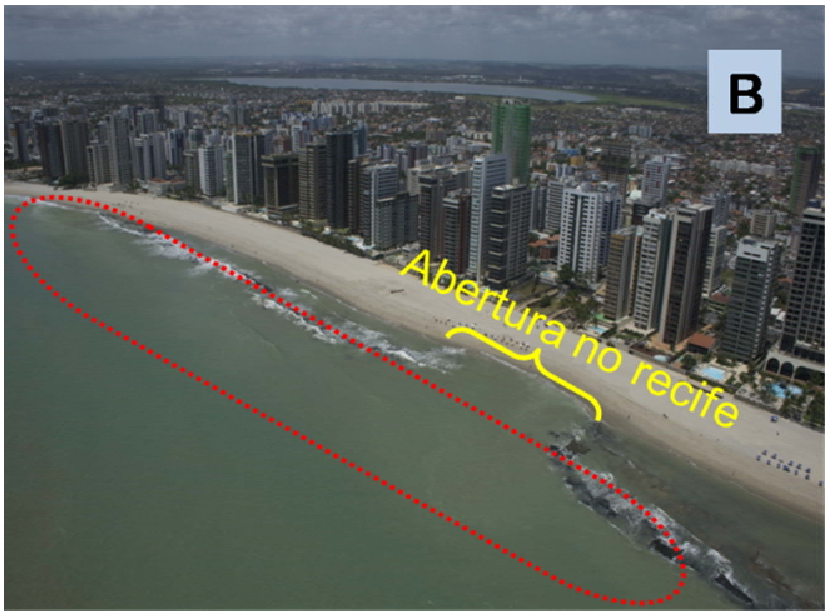


Papel da praia na proteção da costa e as alterações oceanográficas em diferentes escalas temporais

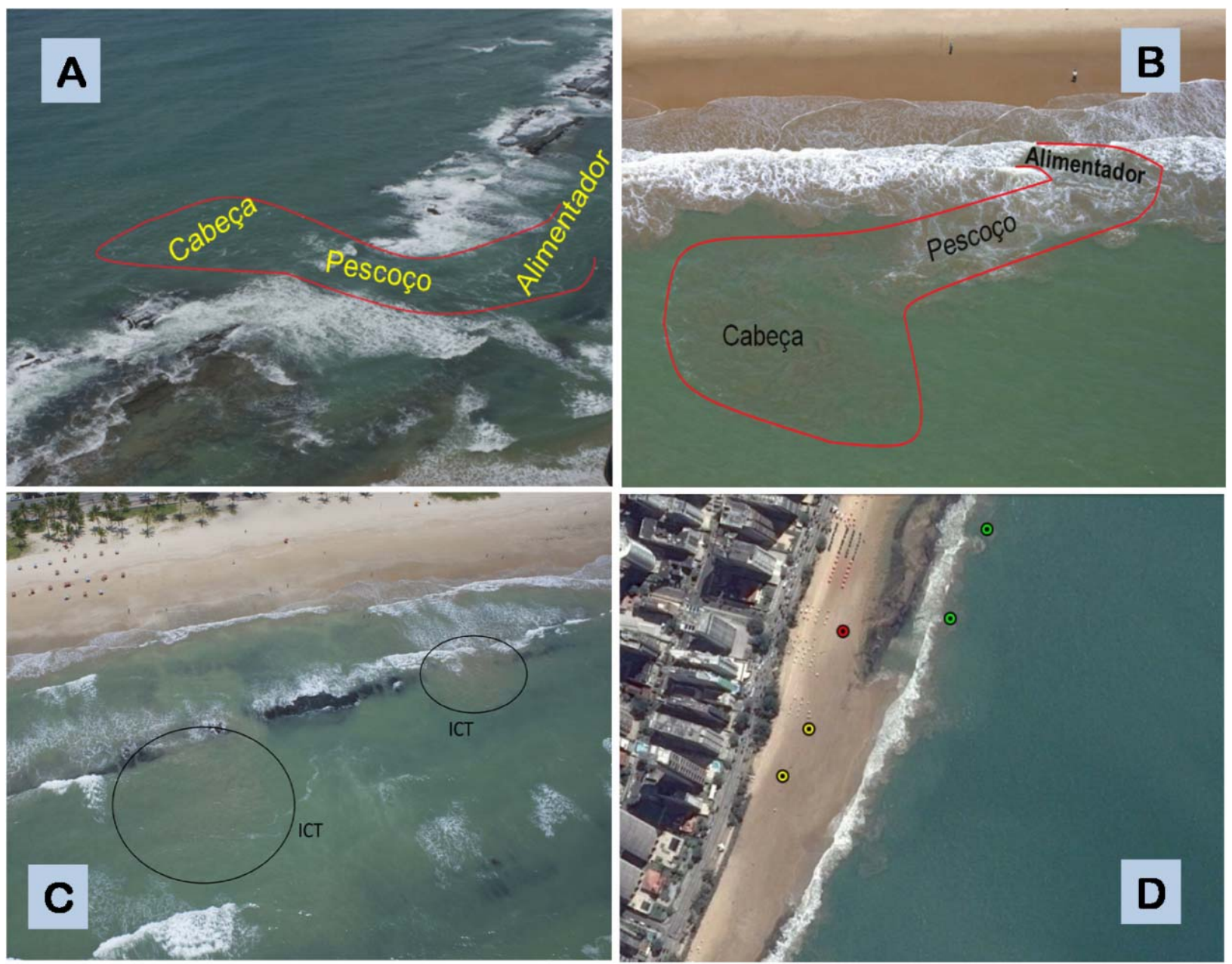

Figura 4: (A) Conformação de uma corrente de retorno fixa; (B) Identificação de uma corrente de retorno móvel; (C) Identificação de um Indicador de Circulação Transversal (ICT); (D) Pontos mapeados.

\section{Mapeamento dos pontos}

Terminada a etapa de identificação de cada corrente de retorno nas imagens, o passo seguinte foi demarcar a posição das mesmas, utilizando imagens de satélite (Google Earth®), empregando o critério de pontos vermelhos representando correntes de retorno fixas, os amarelos representando as correntes móveis e os pontos verdes os Indicadores de Circulação Transversal (ICT), como demonstrado na figura 4c. O termo ICT está sendo aqui proposto devido ao fato de alguns pontos identificados durante o processo de análise das imagens, não possuírem todos os parâmetros - citados anteriormente - que foram usados para a identificação de uma corrente de retorno. Por isso, fez-se o uso do termo indicador, ou seja, havia indícios indicando uma circulação, porém, não possuía os critérios que caracterizasse esse ponto como uma corrente de retorno, propriamente dita. Feições permanentes observadas nas imagens, tais como casas, prédios, estruturas das praias entre outros, serviram para localização desses pontos. Esse mesmo processo foi realizado para as imagens do primeiro e do segundo sobrevôo e para as imagens de satélite do LACCOSTUFPE. Por fim, todos os pontos mapeados e demarcados em cada imagem foram sobrepostos para verificar se dois ou mais pontos coincidiam na localização. Onde ocorreu sobreposição ficou selecionado, como definitivo, o ponto demarcado na imagem de data mais antiga, ou seja, na primeira imagem analisada. Os pontos que não apresentaram uma sobreposição ficaram demonstrando o perfil espaço-temporal de cada município. O número de pontos encontrados foi distribuído espacialmente dentro da região municipal em que ele se encontrava.

\section{Resultados}

Os dois sobrevôos realizados, contemplando os horários de baixamar e preamar, geraram um total de 1.250 imagens aéreas oblíquas. Desse total, 98 delas foram utilizadas para mapeamento das correntes de retorno. Adicionalmente, foi utilizado um conjunto com 11 imagens de satélite, sendo 02 disponibilizadas pelo LACCOST-UFPE e mais 09 imagens do Google Earth ${ }^{\circledR}$. O mapeamento realizado contabilizou 85 fenômenos oceanográficos de circulação da água ocorridos entre zona de surf e linha de praia. Do total de pontos, as correntes de retorno, fixas e móveis, obtiveram um número igual a 77 . O restante, 08 pontos, foram classificados como Indicadores de Circulação Transversal (ICT). 68\% do mapeamento e espacialização foram compostos das imagens do $1^{\circ}$ sobrevôo. Já no $2^{\circ}$ sobrevôo foram espacializados 25 
Papel da praia na proteção da costa e as alterações oceanográficas em diferentes escalas temporais

pontos que representaram $32 \%$ do mapeamento (figura 5).

\section{Mapeamento por sobrevôo}

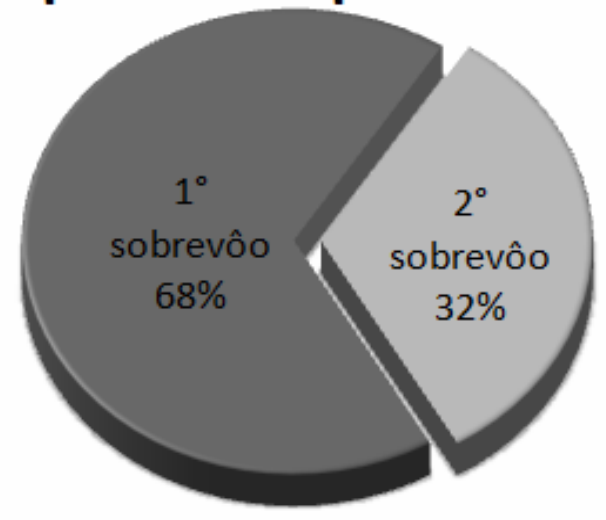

Figura 5: Diagrama do percentual de pontos mapeados em cada sobrevôo realizado.

As duas imagens de satélite do LACCOST, bem como as nove imagens do Google Earth, foram utilizadas para corroborar o mapeamento na área de Piedade até a área do Pina. Dos 15 pontos mapeados nessa porção da área de estudo, 11 foram corroborados por essas imagens. A classificação das correntes de retorno, em toda a área estudada, teve relação com o perfil morfológico encontrado em associação com ela, sendo 52 correntes relacionadas a canais transversais presentes em zonas de surfe ativas, e 23 correntes de retorno em conjunção com estruturas fixas, especificamente com 22 se relacionando a recifes de arenito e 01 relacionada à rocha (figura 6).

A quantidade de CRs mapeadas em cada município tornou possível traçar um perfil da espacialização ao longo da costa da área de estudo, demonstrando os diferentes valores de ocorrência desses fenômenos (figura 7).

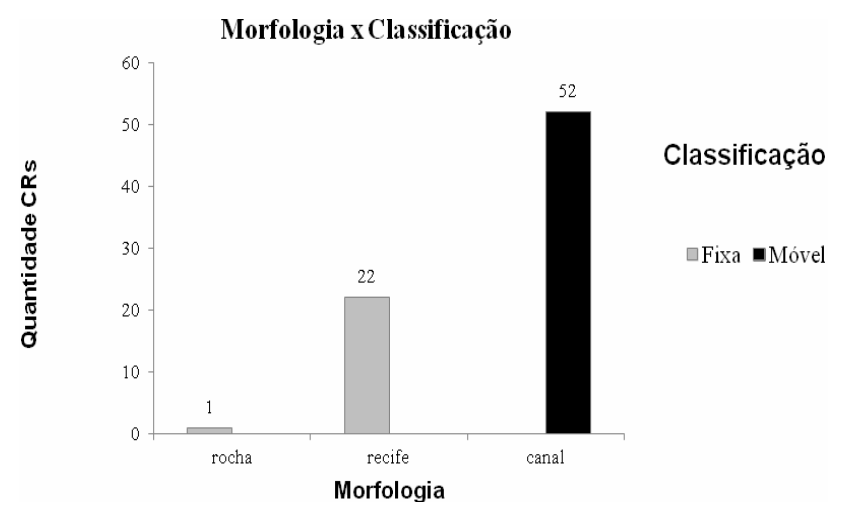

Figura 6: Relação entre morfologia associada à corrente de retorno e classificação denominada.

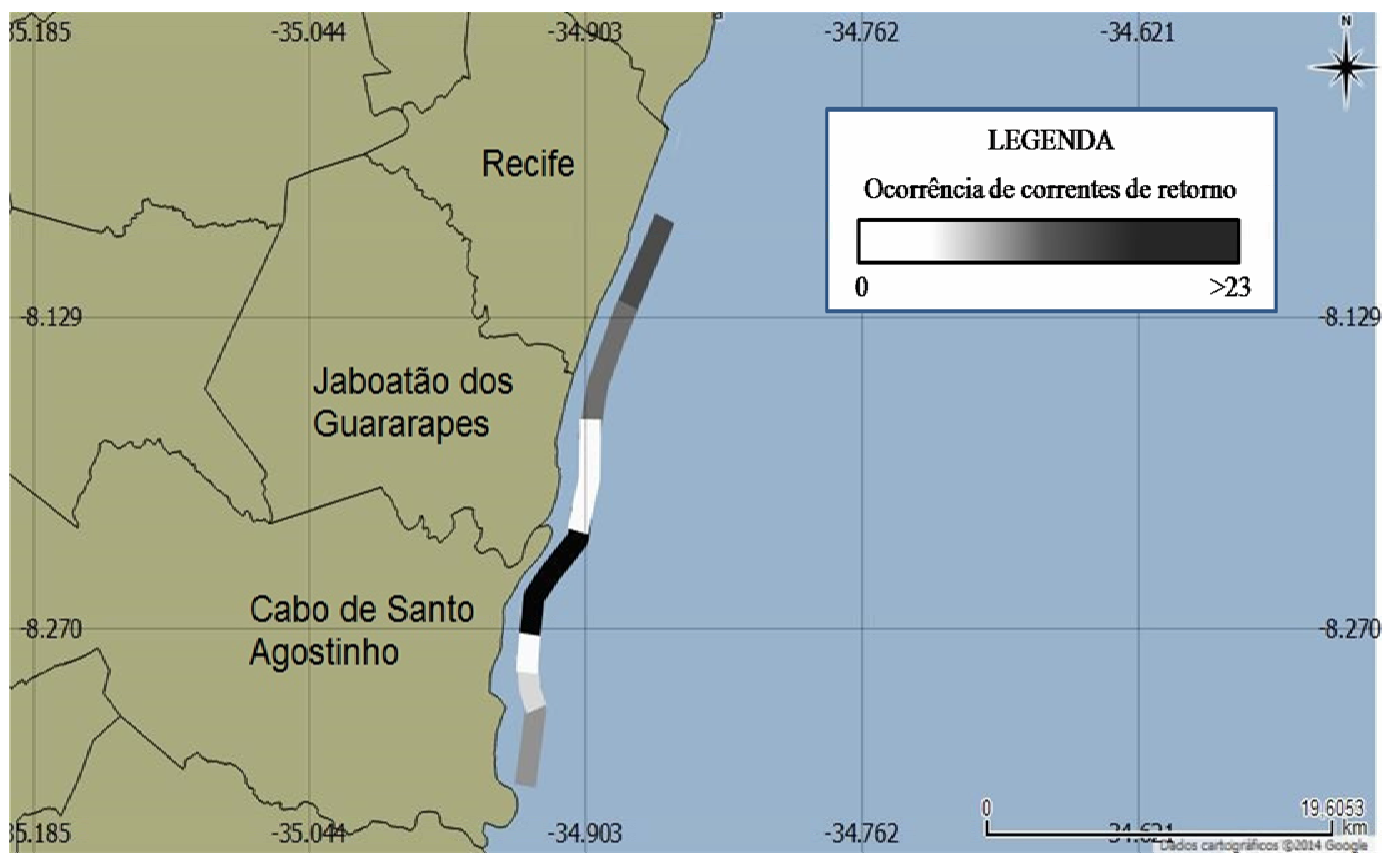

Figura 7: Distribuição da ocorrência de correntes de retorno nos municípios de Recife, Jaboatão dos Guararapes e Cabo de Santo Agostinho.

\section{Município do Cabo de Santo Agostinho}

Na praia de Gaibu apenas uma corrente de retorno foi mapeada, sendo esta classificada como fixa por conta da associação com o recife. Esta mesma corrente, pela relativa proximidade entre as duas praias, foi incorporada a região da praia de Enseada dos Corais afim de melhor incrementar as análises posteriores referentes ao espaçamento das correntes de retorno.
Na área da praia de Enseada dos Corais foi mapeado um total de 08 correntes de retorno, com 05 delas sendo fixas e 03 móveis, além de 02 ICTs. Essa maioria de correntes de retorno fixas se dá, por conta da existência de um recife de arenito que está presente em boa parte da praia, se estendendo até a costa de Gaibu.

Na Praia de Pedra do Xaréu foram demarcadas duas correntes de retorno, com uma sendo fixa e outra 
Papel da praia na proteção da costa e as alterações oceanográficas em diferentes escalas temporais

móvel. A corrente de retorno fixa se caracterizou em conjunção com uma feição morfológica rochosa da própria formação geológica do local.

A praia de Itapuama também apresentou uma forte presença de recife de arenito, fato que teve contribuição para a ocorrência de quatro correntes de retorno fixas, das seis observadas. As demais correntes de retorno foram identificadas como móveis.
A região do Paiva, aqui considerada a reserva do Paiva e Ilha do Amor, foi a que mais apresentou o fenômeno oceanográfico das correntes de retorno, 37 no total. Sendo 23 correntes de retorno para a Reserva do Paiva e 14 para a Ilha do Amor. Assim, a maioria foi identificada como correntes de retorno móveis que possuem como perfil morfológico um canal transversal a zona de surfe (figura 8).

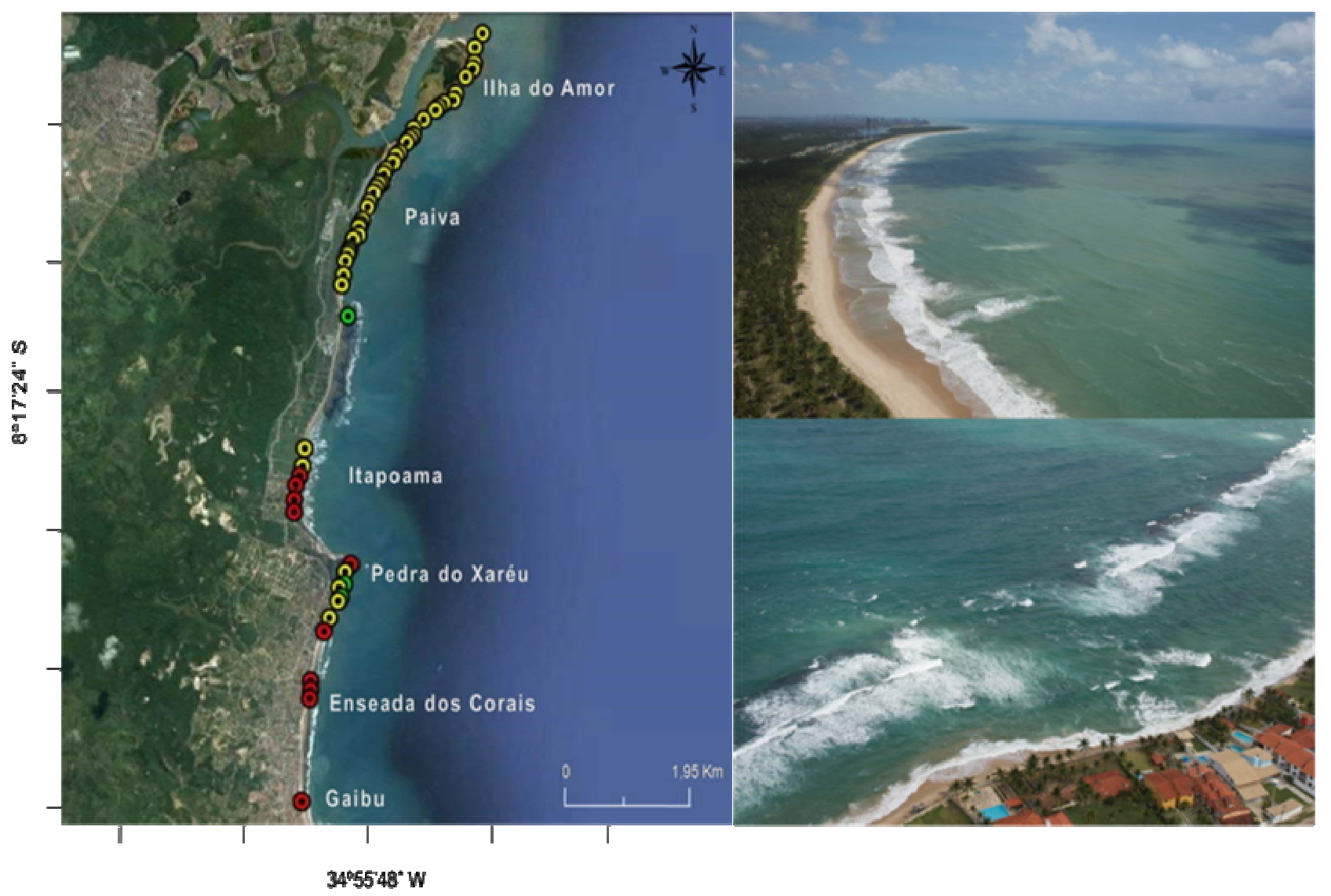

Figura 8: De sul para norte: Enseada dos Corais, Pedra do Xaréu, Itapuama, Paiva e Ilha do Amor. Os pontos em vermelho significam a presença de correntes de retorno fixas, os amarelos móveis e os verdes Indicadores de Circulação Transversal. As imagens oblíquas no lado direito da figura mostram a presença de correntes de retorno móveis na praia do Paiva (imagem superior) e uma corrente de retorno fixa devido a abertura no recife na praia de Enseadas dos Corais (imagem inferior).

\section{Município de Jaboatão dos Guararapes}

O município de Jaboatão é representado pelas praias de Candeias, Barra de Jangada e Piedade, como citado anteriormente. Porém nas duas primeiras praias não foram identificadas correntes de retorno nem ICTs. Já na praia de Piedade foram demarcadas 09 correntes de retorno, com 06 correntes sendo móveis e 03 fixas. Adicionalmente foi demarcado um ICT (figura 9).

\section{Município de Recife}

No município de Recife, que abrange as praias de Boa Viagem e do Pina, foram identificadas 15 correntes de retorno no total e 04 ICTs. Na praia de Boa Viagem foram encontradas 13 correntes de retorno e os 04 Indicadores de Circulação Transversal. Nessa praia também houve predominância de correntes de retorno fixas, num total de 09 correntes. O restante das 04 correntes foi classificado como móveis. A porção da praia do Pina possui um expressivo banco de arrecife, fato esse, que contribui para a presença de duas correntes de retorno fixas nas depressões do mesmo (figura 10). 
Quaternary and Environmental Geosciences (2014) 05(2):166-176

Papel da praia na proteção da costa e as alterações oceanográficas em diferentes escalas temporais

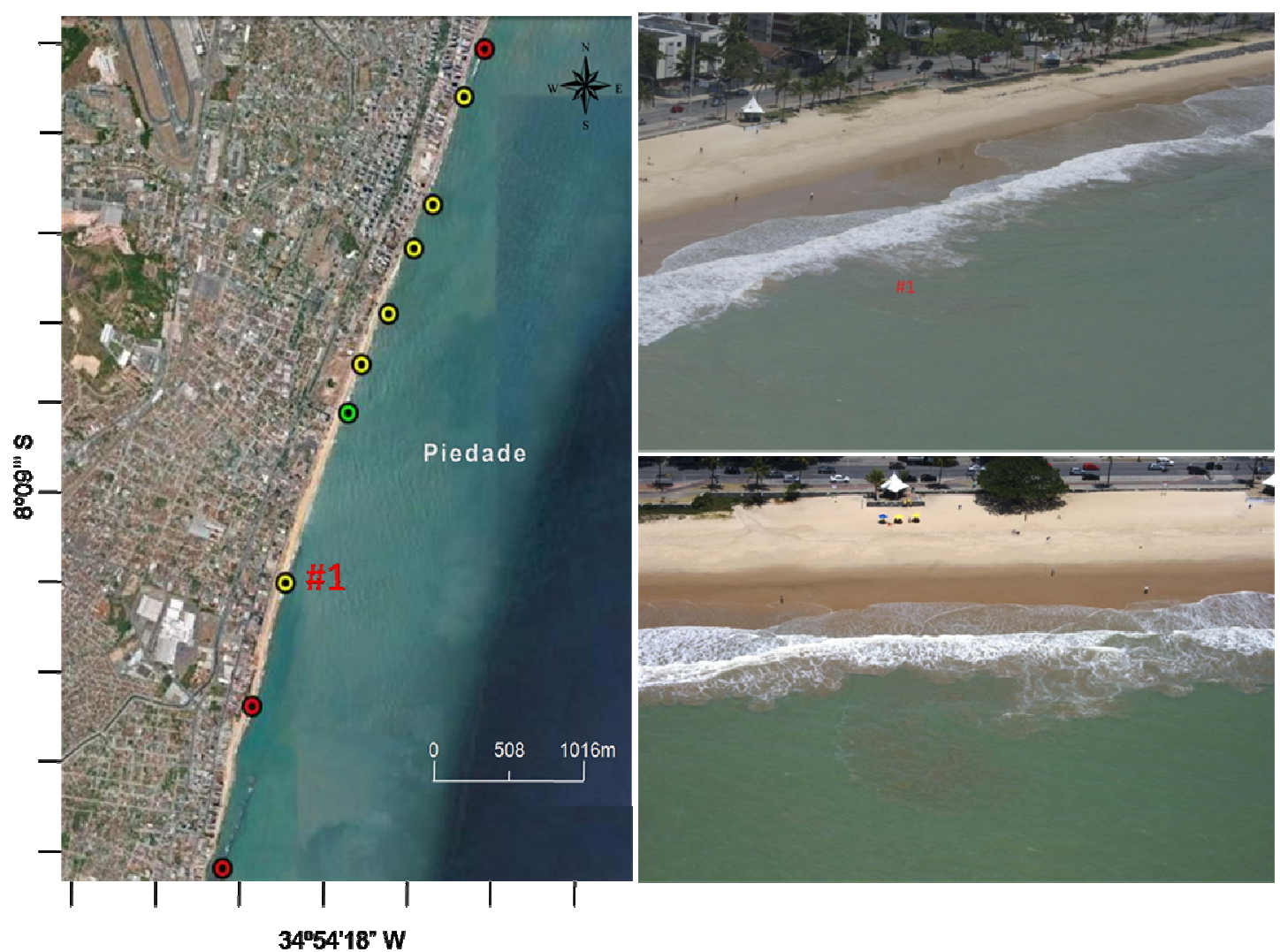

Figura 9: Praia de Piedade. Os pontos vermelhos significam a presença de correntes de retorno fixas, os amarelos móveis e o verde Indicador de Circulação Transversal. As imagens oblíquas no lado direito da figura mostram a presença de uma das correntes de retorno móveis, especificamente a enumerada na figura da esquerda, sendo esta também marcada pelos sedimentos em suspensão.

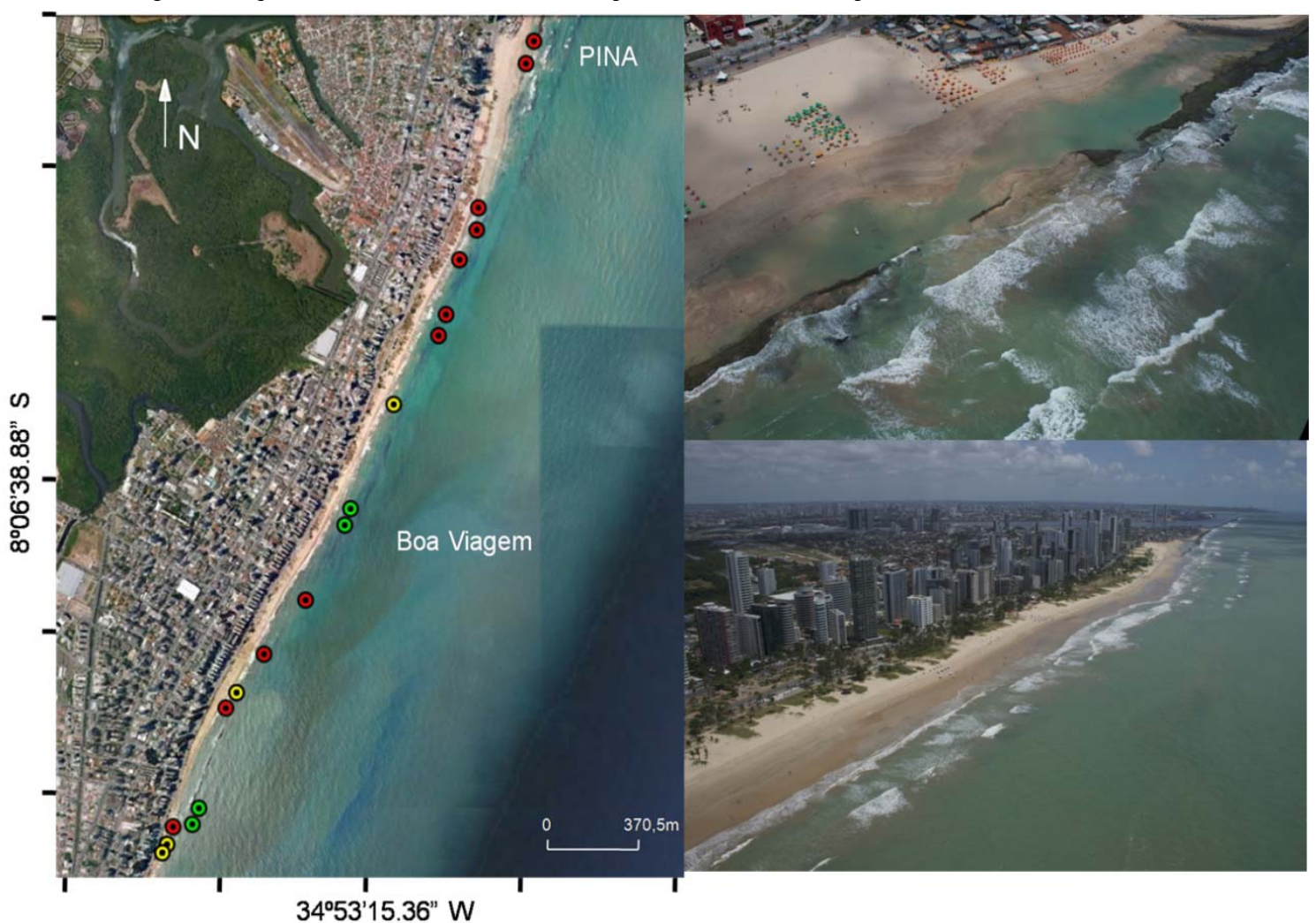

Figura 10: Praia de Piedade. Os pontos vermelhos significam a presença de correntes de retorno fixas, os amarelos móveis e o verde Indicador de Circulação Transversal. As imagens oblíquas no lado direito da figura mostram a presença de correntes de retorno móveis 
Papel da praia na proteção da costa e as alterações oceanográficas em diferentes escalas temporais

\section{Discussão}

A presença de fenômenos oceanográficos, tais como as correntes de retorno, é fortemente influenciada pela morfodinâmica da praia (Calliari et al. 2003). Nesse aspecto a praia do Paiva, por exemplo, possui variados estágios morfodinâmicos. Segundo Tavares et al. (2013) ora ela se encontra em Terraço de Baixa Mar (TBM), ora em Banco e Praia Rítmicos (BPR) ora em Banco Transversal e "Rips” (BTR). Esses estágios morfodinâmicos influenciam na existência de correntes de retorno uma vez que nesses diferentes estágios alguns elementos que tem controle sobre as correntes de retorno, tais como bancos de areia rítmicos e transversais e cavas, também são modificados e/ou não estão presentes (Calliari et al. 2003, Wright \& Short 1984). Este fator é determinante para o perfil móvel das correntes de retorno mapeadas nesta praia. Outro ponto a ser salientado, corroborando os resultados encontrados para o Paiva, é o fato de praias oceânicas abertas tenderem a apresentar CRs transitórias regidas pela energia de grupos de onda que chegam à zona de surfe, nível de maré e pelos fluxos de correntes atuando em ângulo oblíquo e/ou normal a costa gerando células de circulação (Dalrymple et al. 2011, Murray et al. 2013).

Levando em consideração os pontos mapeados como correntes de retorno fixas dentro da área de estudo, pode-se ressaltar o fato de que ao longo da costa de Pernambuco têm-se linhas extensas de recife de arenito, dispostas paralelamente à linha de costa (chegando, em alguns casos, diretamente na praia) que se prolongam, por vezes, continuamente até uma distância de $10 \mathrm{~km}$. Alguns pontos destas linhas afloram na baixa-mar até acima do nível do mar (Araújo 2008). Excetuando a região do Paiva, todas as demais apresentaram correntes de retorno fixas. Para esses pontos se corrobora a ocorrência dessas CRs em depressões dos recifes. Testes laboratoriais já realizados, a fim de se compreender os casos de correntes de retorno associadas a estruturas como recifes (MacMahan et al. 2006), demonstram que a combinação entre altura de onda, seu empilhamento (“setup”) e a depressão do recife, direcionam o fluxo de água para a depressão, criando uma corrente de retorno forte e dependente do estágio da maré. Isso provavelmente se aplica as praias de Gaibu, Enseada dos Corais, Itapuama, Boa Viagem e Pina, praias dos municípios de Cabo de Santo Agostinho e Recife, respectivamente, onde se mapeou 20 correntes de retorno associadas às aberturas no recife de arenito.

O município que teve o menor número de correntes de retorno identificadas foi Jaboatão dos Guararapes, 09 no total, sendo todas elas na área da praia de Piedade. Essa baixa ocorrência de correntes de retorno é baseada no fato dessa praia ser caracterizada como uma praia refletiva e de terraço de baixa-mar uma vez que ela é uma praia abrigada por uma linha de recife (Tavares et al. 2013). Nas demais praias, Barra de Jangada e Candeias, o recife de arenito presente se estende até a linha da costa e isto, possivelmente, acaba forçando as ondas a quebrarem de forma mais efetiva anteriormente a face da praia e não seja possível a formação correntes de retorno, tanto pelo fato de que não há formação de bancos arenosos e canais e nem depressões no próprio recife possibilitando o surgimento do fluxo de água característico das correntes de retorno.

\section{Conclusão}

A orla do Estado de Pernambuco, especificamente a da Região Metropolitana, possui diferenciadas feições e diferenciados estágios praiais. Por conta dessa característica, nas praias dos municípios estudados, foi possível observar a ocorrência das correntes de retorno.

Foi possível, também, diferenciar a quantidade de CRs espacializadas em cada praia e com isso foi possível verificar um perfil diferente para cada município. O município do Cabo de Santo Agostinho foi o que mais apresentou esse tipo de feição oceanográfica, com a praia do Paiva e Ilha do Amor em maior evidência devido ao grande número de correntes de retorno que ocorreram nesta área.

Devido a esse perfil de ocorrência das correntes de retorno em cada município foi possível inferir a maior presença das correntes de retorno móveis com relação às correntes de retorno fixas. Esse aspecto permitiu evidenciar que esse perigo praial pode colocar 0 banhista em situação de perigo, principalmente na praia do Paiva e Ilha do Amor. Para as demais praias essa relação já não tem a mesma proporção, mas mesmo assim representa um alto perigo.

\section{Agradecimentos}

Os autores do presente artigo gostariam de agradecer ao Comitê Estadual de Monitoramento de Incidentes com Tubarões (CEMIT) pelo apoio dado a execução do presente artigo, a Secredaria de Defesa Social do Estado de Pernambuco (SDS) pelos sobrevôos abordo do helicóptero da instituição e ao Laboratório de Cartografia Costeira (LACCOST/UFPE) pela disponibilização das imagens de sátelite de alta resolução para a área de estudo e ao CNPq pela bolsa de Produtividade em Pesquisa (PQ1_Proc: 303251/2010-7) de RPT LESSA.

\section{Referências}

Aagaard T., Greenwood B., Nielsen J. 1997. Mean currents and sediment transport in a rip channel. Mar. Geol. vol.140:24-45.

Albuquerque M. da G., Calliari L.J., Pinheiro L. de S. 2010. Analysis of major risks associated with sea bathing at Futuro beach. Braz. J. Aquat. Sci. Technol., Fortaleza - Ceará. 14(1):1-8.

Araújo M.C. 2008. Praia da Boa Viagem, Recife-PE: análise sócioambiental e proposta de ordenamento. Tese de Doutorado. Programa de Pós-Graduação em Oceanografia. Centro de Tecnologia e Geociências, Universidade Federal de Pernambuco, 279p.

Assis H.M. 1999. Cartografia geomorfológica do Município do Cabo de Santo Agostinho/PE. Recife: CPRM/FIDEM. Série Cartas Temáticas, vol. 04. 32 p.

Assunção P.R.S., Anadir C. da C., Amaral C., Hortência M.B.A, Miranda J.L.F., Pfaltzgraff S.P.A. 1997. Atlas do meio físico do 
Papel da praia na proteção da costa e as alterações oceanográficas em diferentes escalas temporais

Município do Jaboatão dos Guararapes. Estado de Pernambuco. Recife: CPRM/FIDEM, 26p.

Aureliano J.T. 2000. Balneabilidade das praias de Pernambuco o núcleo metropolitano. Dissertação de Mestrado. Programa de Pós-Graduação em Gestão e Políticas Ambientais. Universidade Federal de Pernambuco, 120p.

Borba A.L. 1999. Estudos sedimentológicos, morfodinâmicos e da vulnerabilidade das praias da Piedade, Candeias e Barra de Jangada - Município de Jaboatão dos Guararapes-PE. 1999. Dissertação de Mestrado. Programa de Pós-Graduação em Geociências. Centro de Tecnologia e Geociência, Universidade Federal de Pernambuco, 146p.

Bowman D., Arad D., Rosen D.S., Kit E., Golbery R., Slavicz A. 1988a. Flow characteristics along the rip current system under low-energy conditions. Mar. Geol. 82: 149-167.

Calliari L.J., Toldo Jr. E., Hoefel F. 2003. Morfodinâmica praial: uma breve revisão. Rev. Bras. de Oceanogr., 51: 63 - 78.

Dalrymple R.A., Macmahan J.H., Reniers J.H.M., Nelko V. 2011. Rip Currents. In: Davies S.H., Moin P. (Org.). Annual Review of Fluid Mechanics. Califórnia: Annual Reviews, 551-581.

Holman R.A., Symonds G., Thornton E.B., Ranasinghe R. 2006. Rip spacing on an embayed beach. J. Geophys. Res., 111:17.

Lira L., Hazin F.H., Wor C., Junior H.A. 2010. Estudo de correntes marinhas por meio do lançamento de cartões de deriva no litoral do estado de Pernambuco, Brasil. Arquivos de ciências do mar, Fortaleza, 43(1):30-37.

Macmahan J.H., Thornton E.B., Reniers A.J. 2006. Rip current review. In: Stive M.J. (Org.). Coastal Hydrodynamics and Morphodynamics: Symposium Celebrating the Academic Closing Address of Jurjen A. Battjes. Elsevier. 53(2-3):191-208.

Manso V.V., Coutinho P.N., Guerra N.C., Junior C.F. 2006. Erosão e progradação no litoral brasileiro - Pernambuco. In: Muehe D. (Org.). Erosão e pragradação no litoral brasileiro. Brasília: MMA. 180-196p.

Masselink G., Short A.D. 1993. The effect of tide range on beach morphodynamics and morphology: a conceptual beach model. J. Coast. Res., 9(3): 785 - 800.

Mocellin O. 2006. Determinação do nível de risco público ao banho de mar das praias arenosas do litoral centro norte de Santa Catarina. Dissertação de mestrado. Pós-graduação em Ciência e Tecnologia Ambiental. Centro de Ciências Tecnológicas da Terra e do Mar, Universidade do Vale do Itajaí. 162p.

Munk W.H. 1949a. Surf beats. Trans. Am. Geophys. Union. 30:849854.

Munk W.H., 1949b. The solitary wave theory and application to surf problems. Ann. N. Y. Acad. Sci. 51 (3), 376- 424.

Murray T., Nick C., Rodger T. 2013. Video-imaging of trasient rip currents on the Gold Coast open beaches. In: Conley D.C., Masselink G., Russel P.E., O'Hare T.J. (eds.), Proceedings $12^{\text {th }}$ International Coastal Symposium. Plymouth: Journal of Coastal Research. 65:1809-1814.

Pereira P.S., Calliari L.J., Lélis R.J., Figueiredo S.A. 2003. Riscos associados ao banho de mar e sua relação com a heterogeneidade morfodinâmica das praias do Rio Grande do Sul. In: IX Congresso da Associação Brasileira de Estudos do Quaternário. Recife. 49-61p.

Sasaki T., Horikawa K. 1978. Observation of nearshore current and edge waves. Coastal Engineering. Am. Soc. of Civ. Eng, Reston, Va. 791-809.

Shepard F.P. 1936. Undertow, rip tide, or "rip current". Science. New York City, 84(2173):181-182.

Shepard F.P., Emery K.O., La Fond E.C. 1941. Rip currents: a process of geological importance. J. Geol. 49:337-369.

Shepard F.P., Inman D.L. 1950. Nearshore water circulation related to bottom topography and refraction. Trans. Am. Geophys. Union, 31:196-212.

Short A.D. 1985. Rip-current type, spacing and persistence, Narrabeen Beach, Australia. Marine Geology, Amsterdam, 65:47-71.

Short A.D., Brander R.W. 1999. Regional variations in rip density. J. Coast. Res., 15(3):813-822.

Szpilman D. 2012. Afogamento- Perfil epidemiológico no Brasil. Publicado on-line em www.sobrasa.org, julho de 2012. Brasil.

Tavares I.A., Pereira P.S., Araújo T.C.M., Barcelos R.L. 2013. Variabiliade morfodinâmica da praia do Paiva (Pernambuco,
Brasil). In: XIV Congresso da Associação Brasileira de Estudos do Quaternário, Natal.

Tavares I.A., Pereira P.S., Araújo T.C.M., Barcelos R.L. 2013. Variabiliade morfodinâmica de uma praia abrigada por recife: estudo de caso da praia de Piedade (Pernambuco, Brasil). In: XIV Congresso da Associação Brasileira de Estudos do Quaternário, Natal.

Trujillo A.P., Thurman H.V. 2011. Essentials of oceanography.10 ${ }^{\text {th }}$ ed. Estados Unidos: Prentice Hall. 551p.

Wright L.D., Short A.D.. 1984. Morphodynamics variability of surf zones and beaches: a synthesis. Mar Geol., 56: 93 - 118. .

Recebido 10 de junho de 2014 Aceito 28 de novembro de 2014 